Singular system analysis of the inversion of microwave radiometric data: applications to biological temperature retrieval

This article has been downloaded from IOPscience. Please scroll down to see the full text article. 1987 Inverse Problems 3347

(http://iopscience.iop.org/0266-5611/3/3/006)

View the table of contents for this issue, or go to the journal homepage for more

Download details:

IP Address: 130.251.61.251

The article was downloaded on 21/04/2010 at 09:36

Please note that terms and conditions apply. 


\title{
Singular system analysis of the inversion of microwave radiometric data: applications to biological temperature retrieval
}

\author{
F Bardati†, M Bertero $\ddagger$, M Mongiardo $†$ and D Solimini† \\ † Dipartimento di Ingegneria Elettronica, 2nd University of Rome 'Tor Vergata', 00173 \\ Roma, Italy \\ †Dipartimento di Fisica dell'Universita' and Istituto Nazionale di Fisica Nucleare, 16146 \\ Genova, Italy
}

Received 23 September 1986, in final form 4 December 1986

\begin{abstract}
This paper refers mainly to the inversion of microwave radiometric data for the determination of temperature distributions in the human body. The analysis is restricted to a simple one-dimensional stratified model and special attention is devoted to a skin-fat-muscle structure (SFM) and to a muscle-bone-muscle structure (MBM). In both cases the technique of singular system analysis is applied to the corresponding integral equation in order to quantify the information content of the data. Numerical computations indicate that the information content is much greater in the MвM than in the SFM case, as one can guess from qualitative physical considerations. The results of some numerical inversions are also presented in order to assess the effectiveness and the practicability of the mathematical technique.
\end{abstract}

\section{Introduction}

The relative uniformity of temperature in the bodies of healthy individuals is often altered in diseased organs or affected regions. Both temperature distributions and their variations with time may be characteristics of specific pathologies and may also yield information on the course of an illness [1]. Consequently, biological heat transfer and thermoregulation are of considerable interest to clinicians and physiologists.

In human thermoregulation the heat produced inside the body tissues is balanced by that lost to the environment. The heat exchange processes occur substantially across the skin which is the interface between the inner tissues and the environment. The measure of the skin temperature in itself has proven to give significant indication about the occurring physiological and pathological processes. Infrared thermography has been considered in the past years as an effective method for mapping skin surface temperatures based on the measurement of electromagnetic radiation from the skin at infrared wavelengths [2]. However, non-invasive detection and monitoring of subcutaneous temperature distributions still remains as an important goal in biomedicine. Prospective applications range from the direct detection of deep thermal anomalies to the temperature control during the heating of neoplastic tissues in loco-regional hyperthermia treatment of tumours.

While infrared thermographs measure the thermal emission from the body surface only, microwave radiometers are able to detect the radiation emitted by the inner tissues up to a depth of a few centimetres. In recognition of this potentiality, microwave radiometry has been proposed and experimented to obtain information on the subcutaneous 
temperature distribution in the human body. Laboratory and clinical experimental validation of microwave radiometry has generally been conducted by using singlefrequency systems. Suitable processing of this kind of data has produced significant results, particularly in the detection of left-right asymmetries in breast-cancer cases $[3,4]$. Indications on the temporal thermal variations produced by hyperthermic treatments have also been obtained [5].

Multispectral radiometry has been considered recently as a technique able to provide information about human temperature distributions $[6,7]$ and has already been applied to atmospheric sounding [8]. Indeed, during the past two decades measurements of the thermal emission from the earth's atmosphere in several microwave bands have been used to reconstruct the height distribution (profiles) of variables relevant to meteorological forecasting and monitoring [9]. In spite of the mathematical difficulties encountered in reconstructing the profiles, which are inherent in the kind of equation being solved, nevertheless operational retrievals are nowadays of use in meteorology [10]. The principle on which the radiometric technique is based can be summarised as follows. The contribution to the emission from a given region of the medium under observation varies with frequency. A suitable processing of emission data taken at different frequencies allows the particular contribution from each region to be singled out. At least in principle, therefore, a map of the temperature distribution in the observed medium can be produced from an appropriate set of measurements.

Modelling emission from biological structures is preliminary to the use of the radiometric technique for thermal mapping in the human body. A layered plane parallel model of tissues is adequate to represent the basic electromagnetic behaviour of actual biological structures, at least in cases when the boundaries between the tissues are smooth and the embedded inhomogeneities are negligible. A three-layer structure consisting of skin-fat-muscle (SFM) and muscle-bone-muscle (MBM) arrangements appears to be representative of the essential emitting properties of several regions of the human body. The solution of the thermal emission problem for such biological structures leads to the determination of the radiative transfer kernels, which give the microwave brightness temperature in terms of the thermodynamic temperature distribution within the tissues.

In this paper we are concerned with the inverse problem, i.e. the retrieval of the thermodynamic temperature from measured values of the brightness temperature. To this purpose we first give, in $\S 2$, the solution of the direct problem by providing explicit expressions, derived in previous work, of the radiative transfer kernels (usually called, in radiometry, weighting functions). In such a way the inverse problem can be modelled as the solution of a first-kind Fredholm integral equation. This is a classical example of an illposed problem; the solution is not stable with respect to small variations of the data. This difficulty can be circumvented if one recognises that it is only possible to extract from the data a finite amount of information about the solution, in the sense that only a finite number of components of the solution with respect to a suitable basis can be accurately determined [12-14]. The appropriate basis is provided by the singular system of the integral operator involved in the equation to be solved [15].

In $\S 3$ we give a short description of the case where the brightness temperature is supposed to be known for all the values of the frequency in a suitable band. Then we introduce an approximation of this problem by assuming that the brightness temperature is known for a sufficiently large number of frequencies in the same band. The singular system is computed for the latter problem both in the case of the SFM and in the case of the MBM structure. In such a way the amount of information which can be extracted from the data is quantified in the two cases. Furthermore, we show that the amount of information can be 
enhanced if one restricts, by means of appropriate masks (or 'profile functions'), the region where one intends to recover the physical temperature.

A difficulty inherent to the previous approach is that the singular functions of the problem are discontinuous across the interface between layers. As a consequence the retrieved thermodynamic temperature does not have the correct continuity properties. In $\S 4$ we show that it is possible to avoid this difficulty by introducing a suitable space of continuous functions (Sobolev space) and we compute the new singular system of the problem. This formulation also allows the introduction, in a natural way, of the surface temperature as another datum which can be added to the multispectral radiometric data. Again the amount of information is computed in all these cases.

Finally in $\S 5$ we discuss the retrieval algorithm which consists in a 'windowing' of the singular function expansion of the normal solution [16]. The choice of the window can be determined by a trade-off between numerical stability and compatibility with the data. We present also several examples of restorations of delta functions for the various structures, both in the case of discontinuous and in the case of continuous singular functions. The interpretation of these reconstructions in terms of the impulse response of the system, consisting of the radiometer plus the retrieval algorithm, is also discussed and in such a way resolution limits of the method are determined.

\section{Integral equation for the emission from a layered structure}

In the following, for the sake of simplicity, we shall model the subcutaneous region of tissues as a half-space $z<0$, where both the temperature and the physical properties vary only with the distance $z$ from the body surface. By Fourier analysing the electromagnetic emission of radiation from the thermal body in the lossless half-space $z>0$, the spectrum of the brightness temperature $T_{\mathrm{Bp}}(\omega, \boldsymbol{k})$ is obtained, where $\omega=2 \pi f$ is the angular frequency of a monochromatic radiometric channel which measures the time-averaged electromagnetic flux (proportional to $T_{\mathrm{Bp}}$ ), having polarisation $p$ and propagating at the observation angle defined by $\cos \theta=\hat{\boldsymbol{z}} \cdot \hat{\boldsymbol{k}}(\boldsymbol{k}=\hat{\boldsymbol{k}} \omega / c$, where $c$ is the speed of light in the half-space $z>0$ ).

According to the Rayleigh-Jeans approximation, the contribution to $T_{\mathrm{Bp}}$ of a layer of tissue at depth $z$ and of thickness $\Delta z$ is $T(z) W_{\mathrm{p}}(\omega, k, z) \Delta z$, where $T(z)$ is the thermodynamic temperature and $W_{\mathrm{p}} \Delta z$ is a proportionality factor. By adding partial contributions from all depths $z$, the following equation is obtained in the limit $\Delta z \rightarrow 0$ :

$$
T_{\mathrm{Bp}}(\omega, \boldsymbol{k})=\int_{-D}^{0} W_{\mathrm{p}}(\omega, \boldsymbol{k}, z) T(z) \mathrm{d} z+R_{\mathrm{p}}(\omega, \boldsymbol{k})
$$

where the lower limit of integration, $-D$, indicates the depth within the tissues beyond which the contribution to the brightness becomes negligible, and $R_{\mathrm{p}}(\omega, k)$ is the deep-tissue contribution.

Further, we assume that the region $z<0$ can be described by arrangements of $S$ homogeneous layers separated by planes across which the dielectric constant is discontinuous. The $j$ th layer, of constant permittivity $\varepsilon_{j}$ and thickness $d_{j}$, is limited by planes $z=\zeta_{j-1}$ and $z=\zeta_{j}$, if

$$
\zeta_{j}=-\sum_{l=1}^{j} d_{l} \quad(j=1, \ldots, S) ; \quad \zeta_{0}=0,
$$


It has been shown [11] that the weighting function $W_{\mathrm{p}}(\omega, k, z)$ assumes the following expression inside the $j$ th layer:

$$
\begin{aligned}
W_{\mathrm{p}}=\frac{1}{2} B_{j}\left\{\operatorname{Re}\left[k_{j} y_{j}\right]\left|\exp \left[k_{j}\left(z-\zeta_{j}\right)\right]-\Gamma_{j} \exp \left[-k_{j}\left(z-\zeta_{j}\right)\right]\right|^{2}\right\} \\
\left.+\operatorname{Re}\left[k_{j} y_{j}^{*}\right]\left|\exp \left[k_{j}\left(z-\zeta_{j}\right)\right]-\Gamma_{j} \exp \left[-k_{j}\left(z-\zeta_{j}\right)\right]\right|^{2}\right\}
\end{aligned}
$$

where the coefficients $B_{j}$ are recursively generated by

$B_{j}=B_{j-1}\left|1+\gamma_{j-1}\right|^{2}\left|\exp \left(k_{j} d_{j}\right)+\gamma_{j-1} \Gamma_{j} \exp \left(-k_{j} d_{j}\right)\right|^{-2} \quad j=1, \ldots, S$,

$B_{0}=2 / y_{0}$.

By assuming the region $z<-D$ at uniform temperature $T_{\mathrm{dt}}$, the deep-tissue contribution $R_{\mathrm{p}}$ is

$$
R_{\mathrm{p}}=\frac{1}{2} B_{S}\left|1+\gamma_{S}\right|^{2} \operatorname{Re}\left[y_{S+1}\right] T_{\mathrm{dt}} .
$$

Other symbols in (2.3), (2.4) and (2.5) have the following meaning:

$$
\begin{aligned}
& \Gamma_{j}=\left[\gamma_{j}+\Gamma_{j+1} \exp \left(-2 k_{j+1} d_{j+1}\right)\right]\left[1+\gamma_{j} \Gamma_{j+1} \exp \left(-2 k_{j+1} d_{j+1}\right)\right]^{-1} \\
& (j=0, \ldots, S-1) \\
& \Gamma_{S}=\gamma_{S} \quad \gamma_{j}=\left(y_{j}-y_{j+1}\right)\left(y_{j}+y_{j+1}\right)^{-1} \quad(j=0, \ldots, S) \\
& y_{j} \begin{cases}=k_{j} / i \omega \mu_{0} & \text { for horizontal polarisation } \\
=k_{j} / i \omega \varepsilon_{j} & \text { for vertical polarisation }\end{cases} \\
& k_{j}=\frac{\omega}{c_{0}}\left(\sin ^{2} \theta-\varepsilon_{j} / \varepsilon_{0}\right)^{1 / 2} \quad \operatorname{Im}\left[k_{j}\right]<0 \quad(j=0, \ldots, S+1) .
\end{aligned}
$$

Examples of weighting functions computed for six microwave frequencies and for various arrangements of tissues are shown in figure 1 in the SFM case and in figure 2 in the MBM case, with the direction of observation perpendicular to the layers. Note that the $W_{\mathrm{p}}$ are strongly dependent on frequency and are discontinuous at the interface between different adjacent media.

\section{Singular system analysis}

The inverse problem is the retrieval of the physical temperature $T(z)$ from the measured values of the brightness temperature $T_{\mathrm{Bp}}(\omega, k)$. According to the model described in $\S 2$ this is equivalent to solving equation (2.1) which is a first-kind Fredholm integral equation. In the following we will assume, for simplicity, that the deep-tissue contribution $R_{\mathrm{p}}$ is zero. In a practical situation $R_{\mathrm{p}}$ can be computed and subtracted from the measured brightness temperature. We will also assume a fixed polarisation and a fixed observation angle (for example, $\theta=0$ ); then the brightness temperature is a function of the angular frequency only. We will denote the brightness temperature by $T_{\mathrm{B}}(\omega)$, instead of $T_{\mathrm{Bp}}(\omega, k)$, and the radiative transfer kernel (weighting function) by $W(\omega, z)$, instead of $W_{\mathrm{p}}(\omega, \boldsymbol{k}, z)$. Common values of the frequency are in the band between 1.5 and $6.5 \mathrm{GHz}$. The corresponding lower and upper value of the angular frequency will be denoted by $\omega_{l}$ and $\omega_{u}$, respectively, and we will define $B_{\omega}=\omega_{u}-\omega_{l}$. 


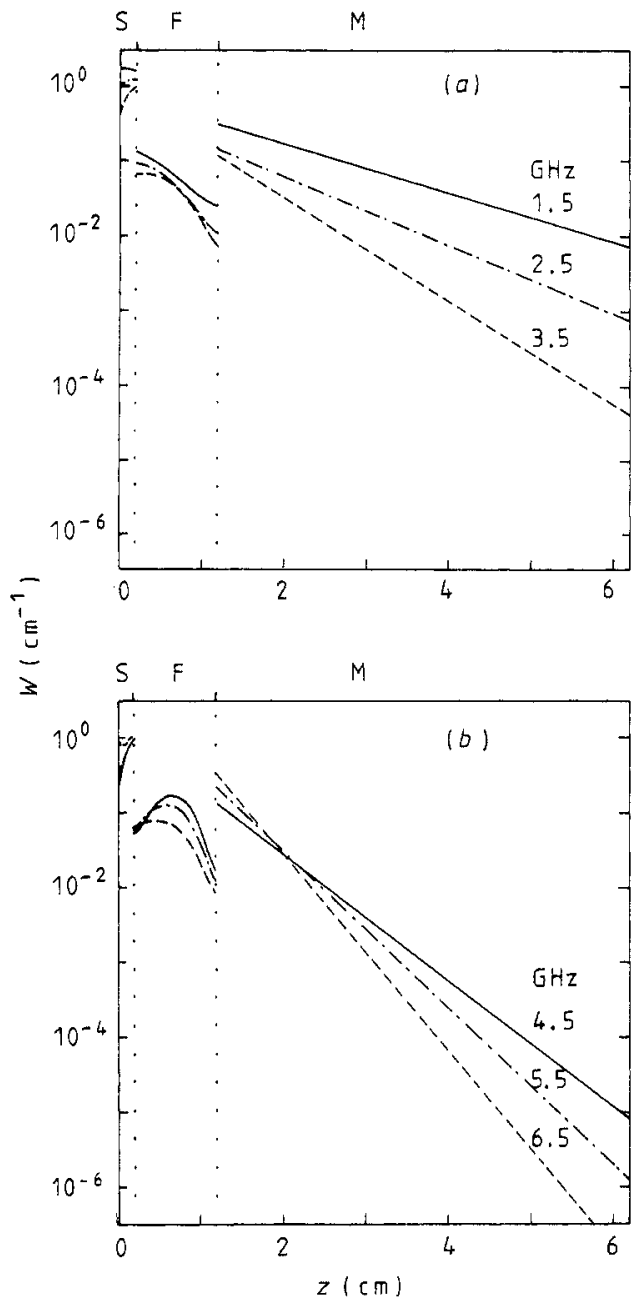

Figure 1. Weighting functions against depth $z$ for the skin-fat-muscle (SFM) structure at the frequencies: (a) $1.5,2.5,3.5 \mathrm{GHz},($ b) $4.5,5.5,6.5 \mathrm{GHz}$.

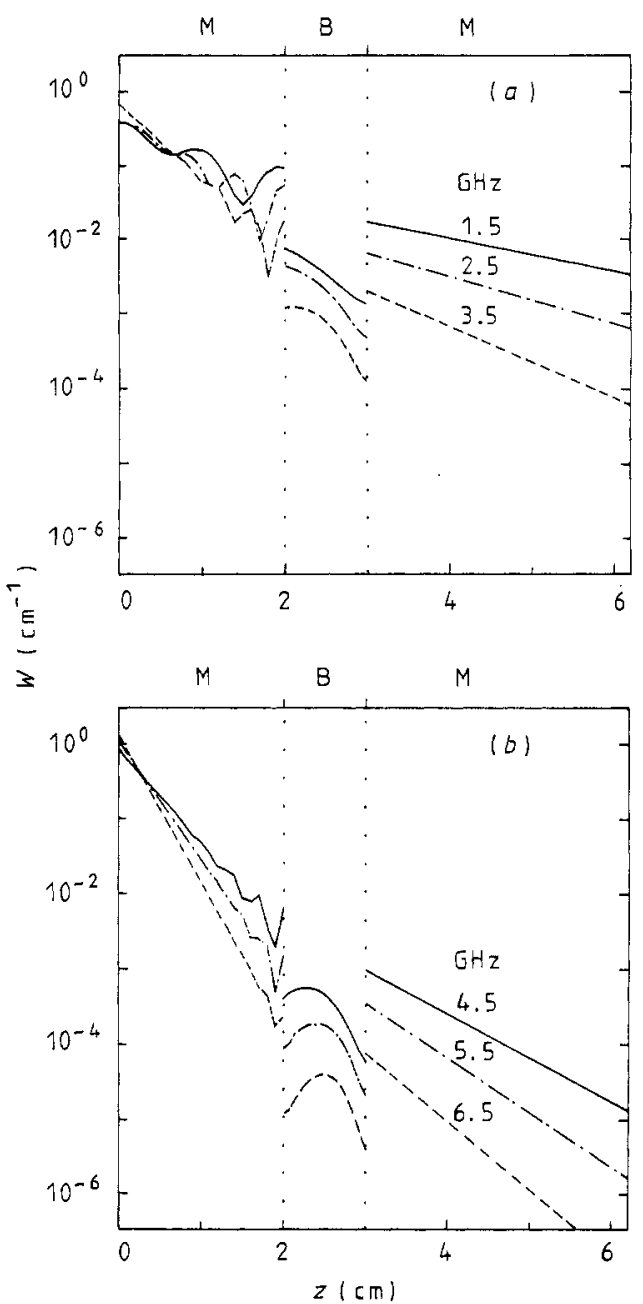

Figure 2. Weighting functions against depth $z$ for the muscle-bone-muscle (MBM) structure at the frequencies (a) $1.5,2.5,3.5 \mathrm{GHz},(b) 4.5,5.5$, $6.5 \mathrm{GHz}$.

The mathematical treatment is considerably simplified if we assume that $T(z)$ is from a space of square-integrable functions, $L^{2}(-D, 0)$, which will be denoted by $X$. In fact $T(z)$ is a bounded function on a bounded interval. We define the norm of $T(z)$ as follows:

$$
\|T\|_{X}=\left(\frac{1}{D} \int_{-D}^{0} T^{2}(z) \mathrm{d} z\right)^{1 / 2}
$$

so that the norm of $T(z)$ has the dimension of a temperature as does $T(z)$. Analogously, the brightness temperature $T_{\mathrm{B}}(\omega)$ can also be assumed to be from a space of square-integrable functions, $L^{2}\left(\omega_{l}, \omega_{u}\right)$, which we shall denote by $Y$, and the norm of $T_{\mathrm{B}}(\omega)$ can also be defined in such a way that it has the dimension of a temperature:

$$
\left\|T_{\mathrm{B}}\right\|_{Y}=\left(\frac{1}{B_{\omega}} \int_{\omega_{l}}^{\omega_{\mu}} T_{\mathrm{B}}^{2}(\omega) \mathrm{d} \omega\right)^{1 / 2} .
$$


Then the integral equation (2.1) defines an integral operator $\mathscr{L}$ from $X$ into $Y$ :

$$
(\mathscr{L} T)(\omega)=\int_{-D}^{.0} W(\omega, z) T(z) \mathrm{d} z \quad \omega_{l} \leqslant \omega \leqslant \omega_{u} .
$$

Since the function $W(\omega, z)$ is bounded and the variables $z, \omega$ can range only over bounded intervals, the operator $\mathscr{L}$ is compact and therefore it admits a singular system $\left.\left\{\alpha_{j} ; u_{j}, v_{j}\right\}\right\}_{j=1}^{\infty}[15]$, which is the set of solutions of the coupled equations

$$
\mathscr{L} u_{j}=\alpha_{j} v_{j} \quad \mathscr{L} * v_{j}=\alpha_{j} u_{j}
$$

where $\mathscr{L}^{*}$ is the adjoint operator

$$
\left(\mathscr{L}^{*} T_{\mathrm{B}}\right)(z)=\frac{D}{B_{\omega}} \int_{\omega_{l}}^{\omega_{u}} W(\omega, z) T_{\mathrm{B}}(\omega) \mathrm{d} \omega \quad-D \leqslant z \leqslant 0 .
$$

Notice that, thanks to the choice of the constants in the norms (3.1) and (3.2), the singular values and singular functions are dimensionless quantities.

The singular functions $u_{j}(z)$ form a basis in $X$ while the singular functions $v_{j}(\omega)$ form a basis in $Y$, and therefore, in order to solve equation (2.1), one can expand $T(z)$ and $T_{\mathrm{B}}(\omega)$ respectively as a series of the $u_{j}(z)$ and of the $v_{j}(\omega)$. It follows that, in the absence of noise, the component of $T_{\mathrm{B}}(\omega)$ with respect to a given $v_{j}(\omega)$ is obtained by multiplying by $\alpha_{j}$ the corresponding component of $T(z)$ with respect to $u_{j}(z)$. However, since the $\alpha_{j}$ tend to zero when $j \rightarrow \infty$ [15] and since the data contain an extra term due to noise, the noise contribution in the components of the data with high $j$ is greater than the signal contribution. As a consequence, for a given signal-to-noise ratio one can extract from the data only a finite number of components of $T(z)$. Such a number is the number of degrees of freedom and it represents the number of independent pieces of information that can be extracted from the data [12-14].

In practice, only a discrete set of measurements is given, which can be generated by measuring the brightness temperature of the biological structure at various frequencies in the band $\left[\omega_{l}, \omega_{u}\right]$. If the number of data is sufficiently large, then the problem with discrete data is a fairly good approximation of the problem with continuous data and the number of degrees of freedom is the same in both cases.

Let $W_{n}(z)$ be the weighting function corresponding to the $n$th experimental point, $W_{n}(z)=W\left(\omega_{n}, z\right)$, and let $g_{n}$ be the measured value of the brightness temperature, $g_{n}=$ $T_{\mathrm{B}}\left(\omega_{n}\right)$; then the problem takes the form

$$
g_{n}=\left(T, D W_{n}\right)_{X} \quad n=1, \ldots, N
$$

the scalar product being that associated with the norm (3.1). Therefore, the problem of estimating $T(z)$, given the data vector $g$, is a typical inverse problem with discrete data [16]. Such a discretisation of a first-kind Fredholm integral equation is also known as the method of moment discretisation [17]. $Y_{N}$ :

Equation (3.6) defines a linear operator $\mathscr{L}_{N}^{\prime}$ from $X$ into an $N$-dimensional vector space

$$
\left(\mathscr{L}_{N} T\right)_{n}=\left(T, D W_{n}\right)_{X} \quad n=1, \ldots, N .
$$

We introduce in $Y_{N}$ a norm which is a discrete version of the norm (3.2)

$$
\|\boldsymbol{g}\|_{Y}=\left(\frac{\delta_{\omega}}{B_{\omega}} \sum_{n=1}^{N} g_{n}^{2}\right)^{1 / 2}
$$


where $\delta_{\omega}$ is the distance between adjacent data points (assumed to be equidistant). Therefore $B_{\omega} / \delta_{\omega}=N-1$.

The adjoint operator $\mathscr{L}^{*}$, which transforms a vector of $Y_{N}$ into a function of $X$, can easily be computed and it is given by (see also $[16,17]$ ):

$$
\left(\mathscr{L}_{N}^{*} g\right)(z)=\frac{D \delta_{\omega}}{B_{\omega}} \sum_{n=1}^{N} g_{n} W_{n}(z) .
$$

Again the singular system $\left\{\alpha_{N, j} ; u_{N, j}, v_{N, j}\right\}_{j=1}^{N}$ of the operator $\mathscr{L}_{N}$ is the set of solutions of the coupled equations

$$
\mathscr{L}_{N} u_{N, j}=\alpha_{N, j} v_{N, j} \quad \mathscr{L}_{N}^{*} v_{N, j}=\alpha_{N, j} u_{N, j}
$$

The singular functions $u_{N, j}$ form a basis in the subspace of $X$ spanned by the functions $D W_{n}(z)$, while the singular vectors $\boldsymbol{v}_{N, j}$ form a basis in the $N$-dimensional vector space $Y$. It is not difficult to prove (see, e.g., [16]) that, when the number of data points in the band $\left[\omega_{l}, \omega_{u}\right]$ tends to infinity, then the singular values and singular functions of the problem with discrete data converge to the corresponding singular values and singular functions of the problem with everywhere-defined data. This result justifies the statement that, when $N$ is sufficiently large, the number of degrees of freedom is the same in the two cases.

As concerns the computation of the singular system of the operator $\mathscr{L}_{N}$, it is important to note that the singular values $\alpha_{N, j}$ are the square roots of the eigenvalues of the Gram matrix (see [16])

$$
[G]_{n m}=\frac{D \delta_{\omega}}{B_{\omega}} \int_{-D}^{0} W_{n}(z) W_{m}(z) \mathrm{d} z
$$

Table 1. Singular values for the SFM and MBM structures in the case of 20 equidistant data

\begin{tabular}{|c|c|c|c|c|}
\hline \multirow[b]{2}{*}{$j$} & \multicolumn{2}{|c|}{ SFM } & \multicolumn{2}{|c|}{ MBM } \\
\hline & $\alpha_{N, j}$ & $\alpha_{N, 1} / \alpha_{N, j}$ & $\alpha_{N, j}$ & $\alpha_{N, 1} / \alpha_{N, j}$ \\
\hline 1 & 1.13417 & 1.0 & 1.04742 & 1.0 \\
\hline 2 & $1.71134 \times 10^{-1}$ & 6.62740 & $2.09011 \times 10^{-1}$ & 5.01131 \\
\hline 3 & $1.12366 \times 10^{-1}$ & $1.00936 \times 10^{1}$ & $7.22674 \times 10^{-2}$ & $1.44937 \times 10^{1}$ \\
\hline 4 & $4.70226 \times 10^{-2}$ & $2.41198 \times 10^{1}$ & $6.09915 \times 10^{-2}$ & $1.71732 \times 10^{1}$ \\
\hline 5 & $9.35535 \times 10^{-3}$ & $1.21233 \times 10^{2}$ & $4.37417 \times 10^{-2}$ & $2.39456 \times 10^{1}$ \\
\hline 6 & $2.22345 \times 10^{-3}$ & $5.10097 \times 10^{2}$ & $3.96285 \times 10^{-2}$ & $2.64310 \times 10^{1}$ \\
\hline 7 & $1.22464 \times 10^{-3}$ & $9.26131 \times 10^{2}$ & $2.67694 \times 10^{-2}$ & $3.91275 \times 10^{1}$ \\
\hline 8 & $9.43119 \times 10^{-4}$ & $1.20258 \times 10^{3}$ & $1.75301 \times 10^{-2}$ & $5.97499 \times 10^{1}$ \\
\hline 9 & $5.25618 \times 10^{-4}$ & $2.15779 \times 10^{3}$ & $1.28796 \times 10^{-2}$ & $8.13239 \times 10^{1}$ \\
\hline 10 & $1.84545 \times 10^{-4}$ & $6.14579 \times 10^{3}$ & $4.60981 \times 10^{-3}$ & $2.27216 \times 10^{2}$ \\
\hline 11 & $4.67091 \times 10^{-5}$ & $2.42817 \times 10^{4}$ & $4.32717 \times 10^{-3}$ & $2.42056 \times 10^{2}$ \\
\hline 12 & $3.19144 \times 10^{-5}$ & $3.55381 \times 10^{4}$ & $7.12464 \times 10^{-4}$ & $1.47014 \times 10^{3}$ \\
\hline 13 & $1.93267 \times 10^{-5}$ & $5.86843 \times 10^{4}$ & $1.04959 \times 10^{-4}$ & $9.97936 \times 10^{3}$ \\
\hline 14 & $6.91872 \times 10^{-6}$ & $1.63928 \times 10^{5}$ & $8.42115 \times 10^{-5}$ & $1.24380 \times 10^{4}$ \\
\hline 15 & $2.12829 \times 10^{-6}$ & $5.32904 \times 10^{5}$ & $3.18187 \times 10^{-5}$ & $3.29184 \times 10^{4}$ \\
\hline 16 & $8.98874 \times 10^{-7}$ & $1.26177 \times 10^{6}$ & $2.04579 \times 10^{-5}$ & $5.11989 \times 10^{4}$ \\
\hline 17 & $3.0349 \mathrm{I} \times 10^{-7}$ & $3.73709 \times 10^{6}$ & $1.19182 \times 10^{-5}$ & $8.78845 \times 10^{4}$ \\
\hline 18 & $2.08996 \times 10^{-7}$ & $5.42678 \times 10^{6}$ & $4.24822 \times 10^{-6}$ & $2.46555 \times 10^{5}$ \\
\hline 19 & $1.22399 \times 10^{-7}$ & $9.26618 \times 10^{6}$ & $7.15354 \times 10^{-7}$ & $1.46420 \times 10^{6}$ \\
\hline 20 & $1.97894 \times 10^{-8}$ & $5.73121 \times 10^{7}$ & $4.72012 \times 10^{-7}$ & $2.21906 \times 10^{6}$ \\
\hline
\end{tabular}
points in the band $1.5-6.5 \mathrm{GHz}$. We give also the numbers $\alpha_{N, 1} / \alpha_{N, j}$ which are related to the stability of the truncated singular function expansions (see $\S 5$ ). 
Table 2. Singular values for the SFM and MBM structures after the introduction of the mask described in the text. Also in this case we have assumed 20 equidistant data points in the band $1.5-6.5 \mathrm{GHz}$.

\begin{tabular}{|c|c|c|c|c|}
\hline \multirow[b]{2}{*}{$j$} & \multicolumn{2}{|c|}{ SFM } & \multicolumn{2}{|c|}{$\mathrm{MBM}$} \\
\hline & $\alpha_{N, j}$ & $\alpha_{N, 1} / \alpha_{N, j}$ & $\alpha_{N, j}$ & $\alpha_{N, 1} / \alpha_{N, j}$ \\
\hline 1 & $2.83741 \times 10^{-1}$ & 1.0 & 1.04729 & 1.0 \\
\hline 2 & $7.49206 \times 10^{-2}$ & 3.78723 & $2.07897 \times 10^{-1}$ & 5.03755 \\
\hline 3 & $1.02786 \times 10^{-2}$ & $2.76051 \times 10^{1}$ & $7.21290 \times 10^{-2}$ & $1.45197 \times 10^{1}$ \\
\hline 4 & $1.96970 \times 10^{-3}$ & $1.44053 \times 10^{2}$ & $6.08836 \times 10^{-2}$ & $1.72015 \times 10^{1}$ \\
\hline 5 & $3.18847 \times 10^{-4}$ & $8.89896 \times 10^{2}$ & $4.36698 \times 10^{-2}$ & $2.39820 \times 10^{1}$ \\
\hline 6 & $5.32037 \times 10^{-5}$ & $5.33311 \times 10^{3}$ & $3.92421 \times 10^{-2}$ & $2.66880 \times 10^{1}$ \\
\hline 7 & $8.85673 \times 10^{-6}$ & $3.20368 \times 10^{4}$ & $2.66198 \times 10^{-2}$ & $3.93425 \times 10^{1}$ \\
\hline 8 & $1.48403 \times 10^{-6}$ & $1.91197 \times 10^{5}$ & $1.75083 \times 10^{-2}$ & $5.98169 \times 10^{1}$ \\
\hline 9 & $2.29981 \times 10^{-7}$ & $1.23376 \times 10^{6}$ & $1.28410 \times 10^{-2}$ & $8.15582 \times 10^{1}$ \\
\hline 10 & $3.62766 \times 10^{-8}$ & $7.82160 \times 10^{6}$ & $4.59167 \times 10^{-3}$ & $2.28085 \times 10^{2}$ \\
\hline 11 & $5.81064 \times 10^{-9}$ & $4.88313 \times 10^{7}$ & $4.32505 \times 10^{-3}$ & $2.42145 \times 10^{2}$ \\
\hline 12 & $4.66849 \times 10^{-9}$ & $6.07779 \times 10^{7}$ & $7.02706 \times 10^{-4}$ & $1.49037 \times 10^{3}$ \\
\hline 13 & $4.27929 \times 10^{-9}$ & $6.63057 \times 10^{7}$ & $8.38914 \times 10^{-5}$ & $1.24839 \times 10^{4}$ \\
\hline 14 & $3.89378 \times 10^{-9}$ & $7.28704 \times 10^{7}$ & $8.09612 \times 10^{-5}$ & $1.29357 \times 10^{4}$ \\
\hline 15 & $3.77013 \times 10^{-9}$ & $7.52603 \times 10^{7}$ & $2.02035 \times 10^{-5}$ & $5.18370 \times 10^{4}$ \\
\hline 16 & $1.83289 \times 10^{-9}$ & $1.54805 \times 10^{8}$ & $4.28740 \times 10^{-6}$ & $2.44272 \times 10^{5}$ \\
\hline 17 & $1.72156 \times 10^{-9}$ & $1.64816 \times 10^{8}$ & $3.90229 \times 10^{-7}$ & $2.68379 \times 10^{6}$ \\
\hline 18 & $1.25264 \times 10^{-9}$ & $2.26514 \times 10^{8}$ & $1.42487 \times 10^{-7}$ & $7.35009 \times 10^{6}$ \\
\hline 19 & $8.17576 \times 10^{-10}$ & $3.47052 \times 10^{8}$ & $1.22437 \times 10^{-8}$ & $8.55372 \times 10^{7}$ \\
\hline 20 & $7.48546 \times 10^{-10}$ & $3.79056^{\circ} \times 10^{8}$ & $9.19176 \times 10^{-9}$ & $1.13938 \times 10^{8}$ \\
\hline
\end{tabular}

and that the singular vectors $v_{N, j}$ are just the eigenvectors of $[G]$ associated with the eigenvalues $\alpha_{N, j}^{2}$. Furthermore, a nice property of the problem under consideration is that the integrals in equation (3.11) can be computed analytically. As a consequence, the singular values and singular vectors can be obtained easily by solving a standard eigenvalue problem. Then the singular functions can be computed using the second equation in (3.10) which, written explicitly, gives

$$
u_{N, j}(z)=\frac{D \delta_{\omega}}{B_{\omega} \alpha_{N, j}} \sum_{n=1}^{N}\left(\boldsymbol{v}_{N, j}\right)_{n} W_{n}(z) .
$$

Here $\left(\boldsymbol{v}_{N, j}\right)_{n}$ denotes the $n$th component of the vector $\boldsymbol{v}_{N, j}$. Notice that, if $\boldsymbol{v}_{N, j}$ is normalised to one with respect to the norm of $Y_{N}$, then $u_{N, j}(z)$, given by (3.12), is automatically normalised to one with respect to the norm of $X$.

An analysis of the singular values has been carried out both for the SFM structure and the MBM structure, the depths of the layers being those indicated in figures 1 and 2 , respectively. Furthermore we have considered the cases $N=20$ and $N=30$ and we have found that 20 points provide a rather good approximation of the largest singular values since, using 30 points, we have found a variation on the third digit only. The number of degrees of freedom of the problem is rather small indeed. The results, in the case of 20 data points, are reported in table 1.

The essential feature which can be derived from these results is that the number of degrees of freedom is greater in the MBM case than in the SFM case. For instance, in the MBM case we have 11 singular values such that $\alpha_{N, 1} / \alpha_{N, j}<10^{3}$, while in the SFM case we have only seven singular values satisfying the same condition. 
This effect can easily be explained as follows. The weighting functions of the SFM structure decay as an exponential in the muscle. Therefore our problem is quite similar to the problem of Laplace transform inversion, in which case the number of degrees of
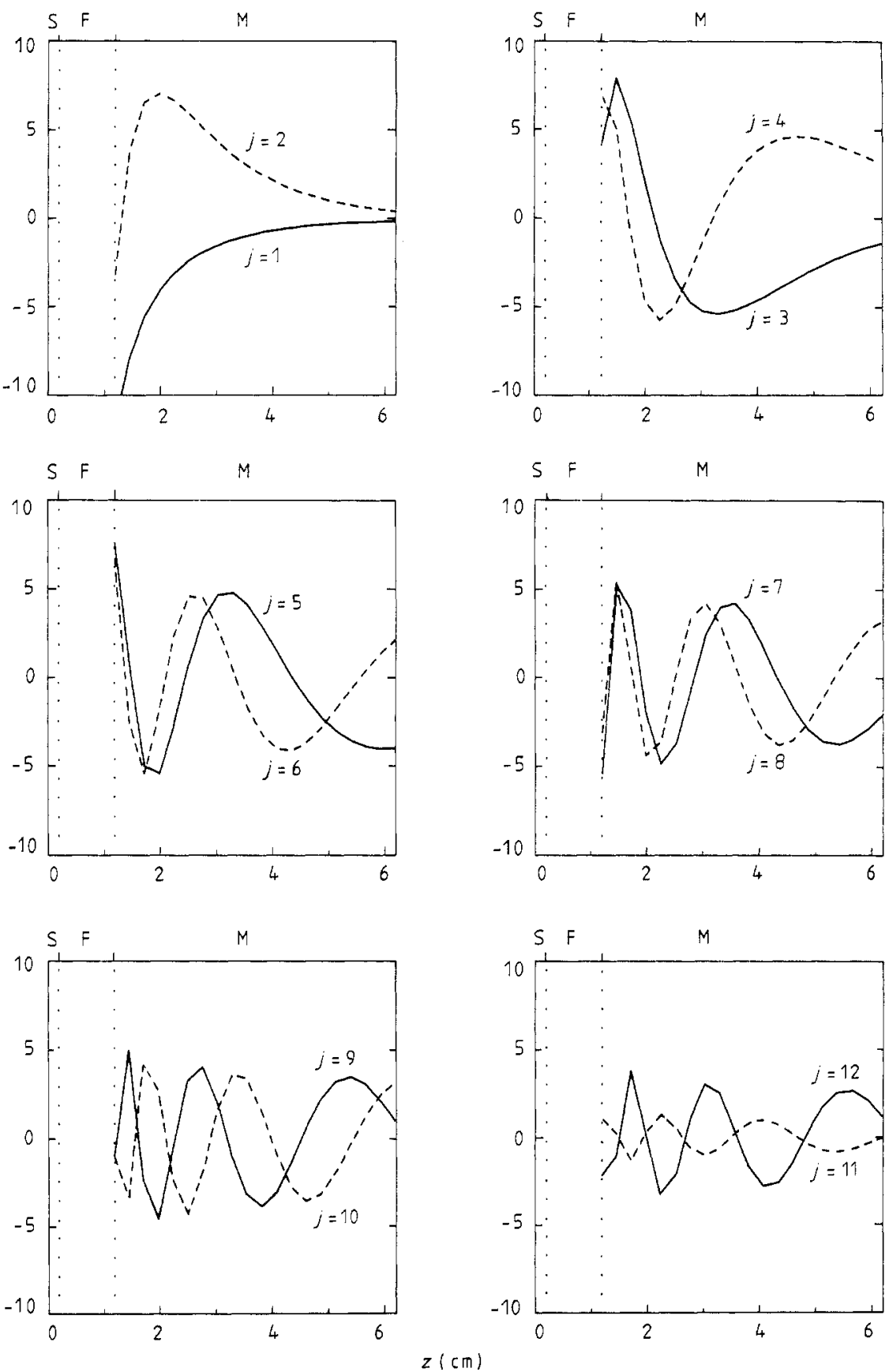

Figure 3. Singular functions $u_{j}$ against depth $z$, with $j=1, \ldots, 12$, for the SFM structure and for a mask which is zero in skin and fat and one in muscle. 
freedom is quite small [18]. On the other hand, in the case of the MBM structure, the weighting functions do not have an exponential decay in the outer muscle, since radiation is reflected by the bone. Therefore it is quite natural to expect that this effect of 'standing
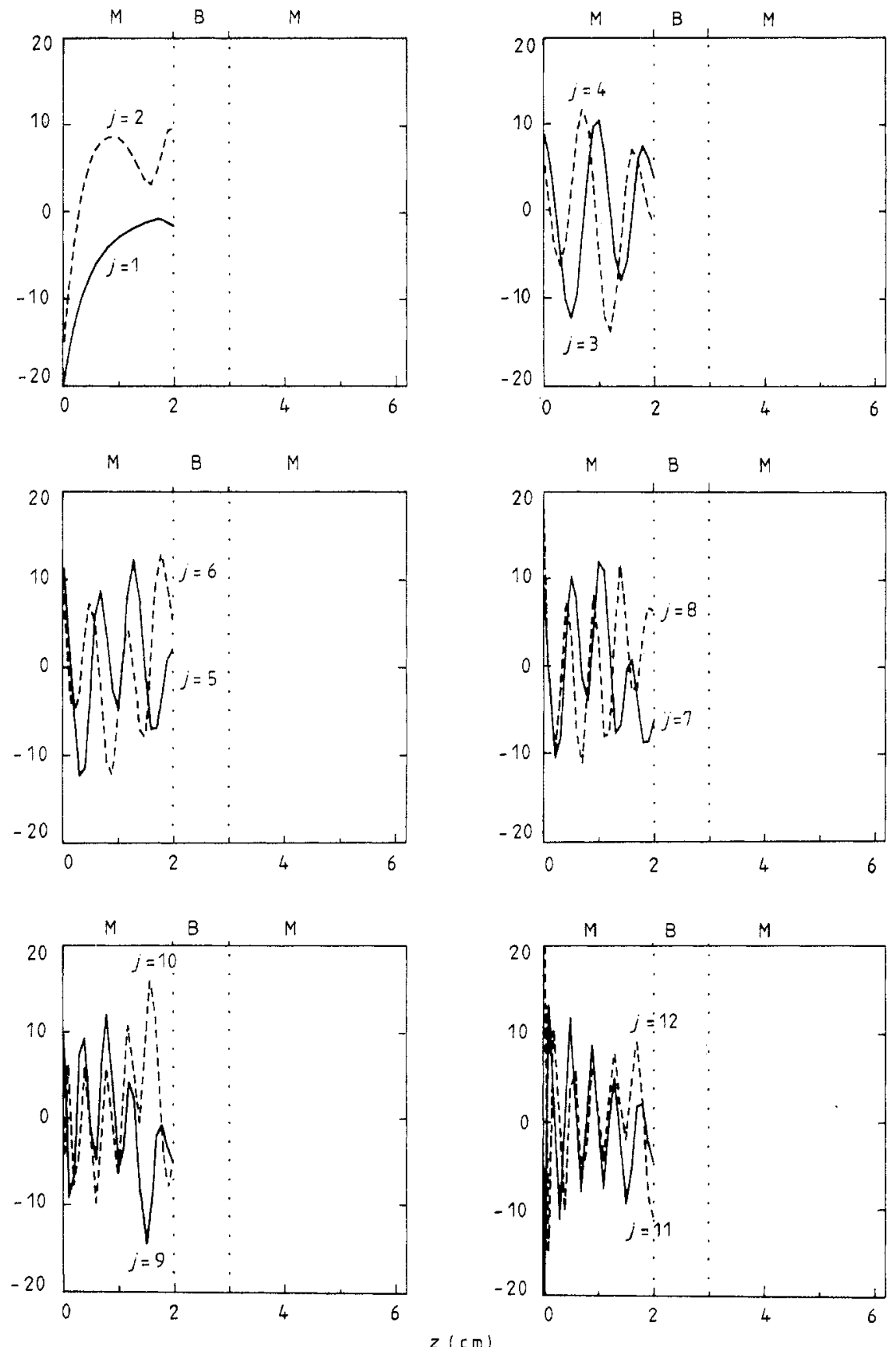

Figure 4. Singular functions $u_{j}$ against depth $z$, with $j=1, \ldots, 12$, for the MBM structure and for a mask which is one in the outer muscle $(0 \leqslant z \leqslant 2 \mathrm{~cm})$ and zero elsewhere. 
waves' in the outer muscle produces an increase of the number of degrees of freedom. This conjecture is confirmed by the results reported in table 1 .

Since the number of degrees of freedom is rather small and consequently resolution is rather poor, one can try to improve the situation in the following way. In the case of the SFM structure one is interested essentially in reconstructing the temperature profile in the muscle, while in the case of the MBM structure it is reasonable to infer that the temperature profile can be reconstructed with reasonable accuracy only in the outer muscle. Therefore in the SFM case we introduce a mask (or a 'profile function') which is zero in the skin and fat and one in the muscle, while in the MBM case we introduce a mask which is one in the outer muscle and zero elsewhere. Obviously the introduction of such a mask is equivalent to integrating, in equation (2.1), only on the muscle in the SFM case and only on the outer muscle in the Mвм case. For a discussion of the improvement in resolution which can be obtained in similar inverse problems by restricting a priori the region where the solution is reconstructed see $[18-20]$.

The results of the computation of the singular values, after the introduction of the previously described masks, are reported in table 2 . Notice that in the case of the MBM structure the singular values greater than $10^{-3}$ essentially coincide with the singular values given in table 1 . This means that the contribution of the bone and of the inner muscle to the significant singular values is negligible and that one can extract information only about the temperature profile in the outer muscle. In any case one can say that the number of degrees of freedom per unit length is increased with respect to the case without mask.

In the case of the SFM structure we have now five singular values such that $\alpha_{N, 1} / \alpha_{N, j}>10^{3}$ (there were seven in the case without mask). But now this amount of information refers only to the muscle.

The singular functions $u_{j}(z)$ are plotted in figure 3 in the SFM case and in figure 4 in the MBM case. From figure 3 we expect a better resolution in the outer part of the muscle since the singular functions are rather flat in the inner region. On the other hand, from figure 4 we expect that, in the мвм case, resolution is roughly uniform over the whole outer muscle. These conclusions will be confirmed in $\S 5$.

\section{Continuity of the solution}

A difficulty in the previous analysis is that the singular functions $u_{j}(z)$ are discontinuous as a consequence of the discontinuities of the weighting functions $W_{n}(z)$. It follows that the restored temperature profile which can be obtained by means of these singular functions does not satisfy some physical requirements. The physical temperature $T(z)$ must be continuous indeed and such that $K(z) T^{\prime}(z)$ is also continuous, $K(z)$ being the thermal conductivity. Notice that $K(z)$ is discontinuous across the interface between layers.

The previous remark leads one to consider a class of functions having the correct regularity properties and to introduce in such a class a suitable scalar product. If we denote by $\bar{X}$ the class of continuous functions we consider, then the scalar product in such a class will be defined by

$$
\begin{aligned}
(T, \Phi)_{\bar{X}}=T(0) \Phi(0)+T(-D) \Phi(-D)+(1 / H) \int_{-D}^{0} B(z) T(z) \Phi(z) \mathrm{d} z \\
+(1 / H) \int_{-D}^{0} K(z) T^{\prime}(z) \Phi^{\prime}(z) \mathrm{d} z
\end{aligned}
$$


where the function $B(z)$ and the constant $H$ will be specified on the basis of physical considerations. In any case the dimensions of these quantities are determined by the requirement that the norm of $T(z)$, defined by $\|T\|_{\bar{X}}=(T, T)_{\bar{X}}^{1 / 2}$, has the dimension of temperature. In the jargon of functional analysis, a space of continuous functions, characterised by a scalar product as in (4.1), is called a Sobolev space.

In this section we focus essentially on the problem with discrete data. Then, if we want to formulate the problem (3.6) in the new space, we must look for functions $\Phi_{n}(z)$ such that, for any $T(z)$,

$$
\left(T, \Phi_{n}\right)_{\bar{X}}=\int_{-D}^{0} T(z) W_{n}(z) \mathrm{d} z .
$$

By means of a partial integration in the second integral of equation (4.1), it is easy to see that, for a given $W_{n}$, the corresponding $\Phi_{n}$ must be solutions of the following boundary value problem:

$$
\begin{aligned}
& -\left[K(z) \Phi_{n}^{\prime}(z)\right]^{\prime}+B(z) \Phi_{n}(z)=H W_{n}(z) \\
& K(0) \Phi_{n}^{\prime}(0)+H \Phi_{n}(0)=0 \\
& -K(-D) \Phi_{n}^{\prime}(-D)+H \Phi_{n}(-D)=0
\end{aligned}
$$

It follows that the functions $\Phi_{n}$ have a very nice physical interpretation. In fact, equation (4.3) describes a stationary linear heat flow in a one-dimensional solid layered along the $z$ axis when heat is supplied at the rate $H W_{n}(z)$ per unit volume. In this analogy, $\Phi_{n}(z)$ is the temperature distribution and $B(z) \Phi_{n}(z)$ represents a distributed linear heat transfer between the solid and a cooling fluid at zero temperature. Linear heat transfer on the boundary of the solid into a surrounding medium at zero temperature is taken into account by the boundary conditions (4.4) and (4.5). When the thermal behaviour of electromagnetically irradiated living tissues is considered, the above differential equation is obtained through linearisation of an appropriate nonlinear problem [21].

From equation (4.2) it follows that the inverse problem now takes the following form:

$$
g_{n}=\left(T, \Phi_{n}\right)_{\bar{X}} \quad n=1, \ldots, N .
$$

If in the data space $Y_{N}$ we take again the norm (3.8), we can define, by a relation similar to (3.7), a linear operator $\overline{\mathscr{L}}_{N}$ which transforms a continuous function of the Sobolev space $\bar{X}$ into a vector of $Y_{N}$. Then the adjoint operator $\overline{\mathscr{L}}_{N}^{*}$ is given by an expression similar to (3.9), with functions $D W_{n}(z)$ replaced by functions $\Phi_{n}(z)$.

The singular system $\left\{\bar{\alpha}_{N, j} ; \bar{u}_{N, j}, \bar{v}_{N, j}\right\}_{j=1}^{N}$ of the operator $\overline{\mathscr{L}}_{N}$ is again the set of the solutions of the coupled equations analogous to equations (3.10) and the singular values $\bar{\alpha}_{N, j}$ with the corresponding singular vectors $\bar{v}_{N, j}$ can be obtained by diagonalising the Gram matrix. The singular functions $\bar{u}_{N, j}$ can be computed successively by means of equation (3.12), with functions $D W_{n}(z)$ replaced again by functions $\Phi_{n}(z)$. Therefore the singular functions $\bar{u}_{N, j}$ satisfy the physical requirements stated at the beginning of this section. If $\bar{v}_{N, j}$ is normalised to one with respect to the norm (3.8), then $\bar{u}_{N, j}$ is automatically normalised to one with respect to the norm induced by the scalar product (4.1).

As in the case of $\S 3$, the crucial point is the computation of the Gram matrix of the basic functions, i.e. the $\Phi_{n}$ in the present case. By means of a partial integration and by taking equations (4.3)-(4.5) into account, one can easily find the following expression for 
the elements of the Gram matrix:

$$
[G]_{n m}=\frac{\delta_{\omega}}{B_{\omega}}\left(\Phi_{n}, \Phi_{m}\right) \tilde{X}=\frac{\delta_{\omega}}{B_{\omega}} \int_{-D}^{.0} \Phi_{n}(z) W_{m}(z) \mathrm{d} z .
$$

For simplicity, we have made preliminary computations in the case $K(z)=$ constant and $B(z)=0$. In such a case the first derivative of $\Phi_{n}(z)$ is continuous and the solution of the boundary value problem (4.3)-(4.5) is given by

$$
\Phi_{n}(z)=\Phi_{n}(0)-h p_{n}(0)+h\left[\tau_{n}(0)-\Phi_{n}(0)\right] z+h p_{n}(z)
$$

where $h=H / K$,

$$
\Phi_{n}(0)=\frac{1}{2+h D}\left[(1+h D) \tau_{n}(0)+h p_{n}(0)\right]
$$

and

$$
\begin{aligned}
& p_{n}(z)=-\int_{-D}^{\cdot z} \tau_{n}(\zeta) \mathrm{d} \zeta \\
& \tau_{n}(z)=\int_{-D}^{z z} W_{n}(\zeta) \mathrm{d} \zeta .
\end{aligned}
$$

It is remarkable also that in such a case the matrix elements $[G]_{n m}$ can be computed analytically.

\begin{tabular}{|c|c|c|c|c|}
\hline \multirow[b]{2}{*}{$j$} & \multicolumn{2}{|c|}{ SFM } & \multicolumn{2}{|c|}{ MBM } \\
\hline & $\dot{\alpha}_{N, j}$ & $\bar{\alpha}_{N, 1} / \bar{\alpha}_{N, j}$ & $\dot{\alpha}_{N, j}$ & $\bar{\alpha}_{N, 1} / \bar{\alpha}_{N, j}$ \\
\hline 1 & $3.16719 \times 10^{-1}$ & 1.0 & $3.56850 \times 10^{-1}$ & 1.0 \\
\hline 2 & $1.75218 \times 10^{-2}$ & $1.80756 \times 10^{1}$ & $2.43332 \times 10^{-2}$ & $1.46652 \times 10^{1}$ \\
\hline 3 & $7.24028 \times 10^{-3}$ & $4.37440 \times 10^{1}$ & $3.46730 \times 10^{-3}$ & $1.02919 \times 10^{2}$ \\
\hline 4 & $1.37360 \times 10^{-3}$ & $2.30575 \times 10^{2}$ & $1.94552 \times 10^{-3}$ & $1.83421 \times 10^{2}$ \\
\hline 5 & $4.71909 \times 10^{-4}$ & $6.71143 \times 10^{2}$ & $1.34612 \times 10^{-3}$ & $2.65094 \times 10^{2}$ \\
\hline 6 & $9.45811 \times 10^{-5}$ & $3.34865 \times 10^{3}$ & $7.77780 \times 10^{-4}$ & $4.58805 \times 10^{2}$ \\
\hline 7 & $7.22917 \times 10^{-5}$ & $4.38112 \times 10^{3}$ & $5.28237 \times 10^{-4}$ & $6.75548 \times 10^{2}$ \\
\hline 8 & $3.01437 \times 10^{-5}$ & $1.05070 \times 10^{4}$ & $2.64253 \times 10^{-4}$ & $1.35041 \times 10^{3}$ \\
\hline 9 & $1.14012 \times 10^{-5}$ & $2.77794 \times 10^{4}$ & $2.08143 \times 10^{-4}$ & $1.71445 \times 10^{3}$ \\
\hline 10 & $4.91230 \times 10^{-6}$ & $6.44746 \times 10^{4}$ & $9.01662 \times 10^{-5}$ & $3.95769 \times 10^{3}$ \\
\hline 11 & $2.84491 \times 10^{-6}$ & $1.11328 \times 10^{5}$ & $4.12319 \times 10^{-5}$ & $8.65471 \times 10^{3}$ \\
\hline 12 & $7.67955 \times 10^{-7}$ & $4.12418 \times 10^{5}$ & $2.01033 \times 10^{-5}$ & $1.77508 \times 10^{4}$ \\
\hline 13 & $3.52857 \times 10^{-7}$ & $8.97583 \times 10^{5}$ & $6.78697 \times 10^{-6}$ & $5.25786 \times 10^{4}$ \\
\hline 14 & $2.65054 \times 10^{-7}$ & $1.19492 \times 10^{6}$ & $1.87767 \times 10^{-6}$ & $1.90049 \times 10^{5}$ \\
\hline 15 & $7.02268 \times 10^{-8}$ & $4.50994 \times 10^{6}$ & $8.50589 \times 10^{-7}$ & $4.19532 \times 10^{5}$ \\
\hline 16 & $4.11756 \times 10^{-8}$ & $7.69191 \times 10^{6}$ & $5.76990 \times 10^{-7}$ & $6.18468 \times 10^{5}$ \\
\hline 17 & $5.01324 \times 10^{-9}$ & $6.31764 \times 10^{7}$ & $3.62576 \times 10^{-7}$ & $9.84208 \times 10^{5}$ \\
\hline 18 & $2.17403 \times 10^{-9}$ & $1.45682 \times 10^{8}$ & $1.32891 \times 10^{-7}$ & $2.68528 \times 10^{6}$ \\
\hline 19 & $1.28859 \times 10^{-9}$ & $2.45787 \times 10^{8}$ & $1.87396 \times 10^{-8}$ & $1.90426 \times 10^{7}$ \\
\hline 20 & $6.44805 \times 10^{-10}$ & $4.91185 \times 10^{8}$ & $1.03619 \times 10^{-8}$ & $3.44387 \times 10^{7}$ \\
\hline
\end{tabular}

In table 3 we give the singular values $\bar{\alpha}_{N, j}$ for the same structures and the same data points considered in $\S 3$. A comparison with table 2 shows that the number of degrees of

Table 3. Singular values for the SFM and MBM structures, in the case of 20 equidistant data points in the band $1.5-6.5 \mathrm{GHz}$, introducing continuity of the temperature distribution. 
freedom is smaller in the present case. The reason is that, by introducing continuity properties, we have strongly restricted the class of the admissible solutions [12]. In figure 5 we plot the singular functions $\bar{u}_{N, j}$ in the SFM case while the singular functions in the MBM case are plotted in figure 6 .
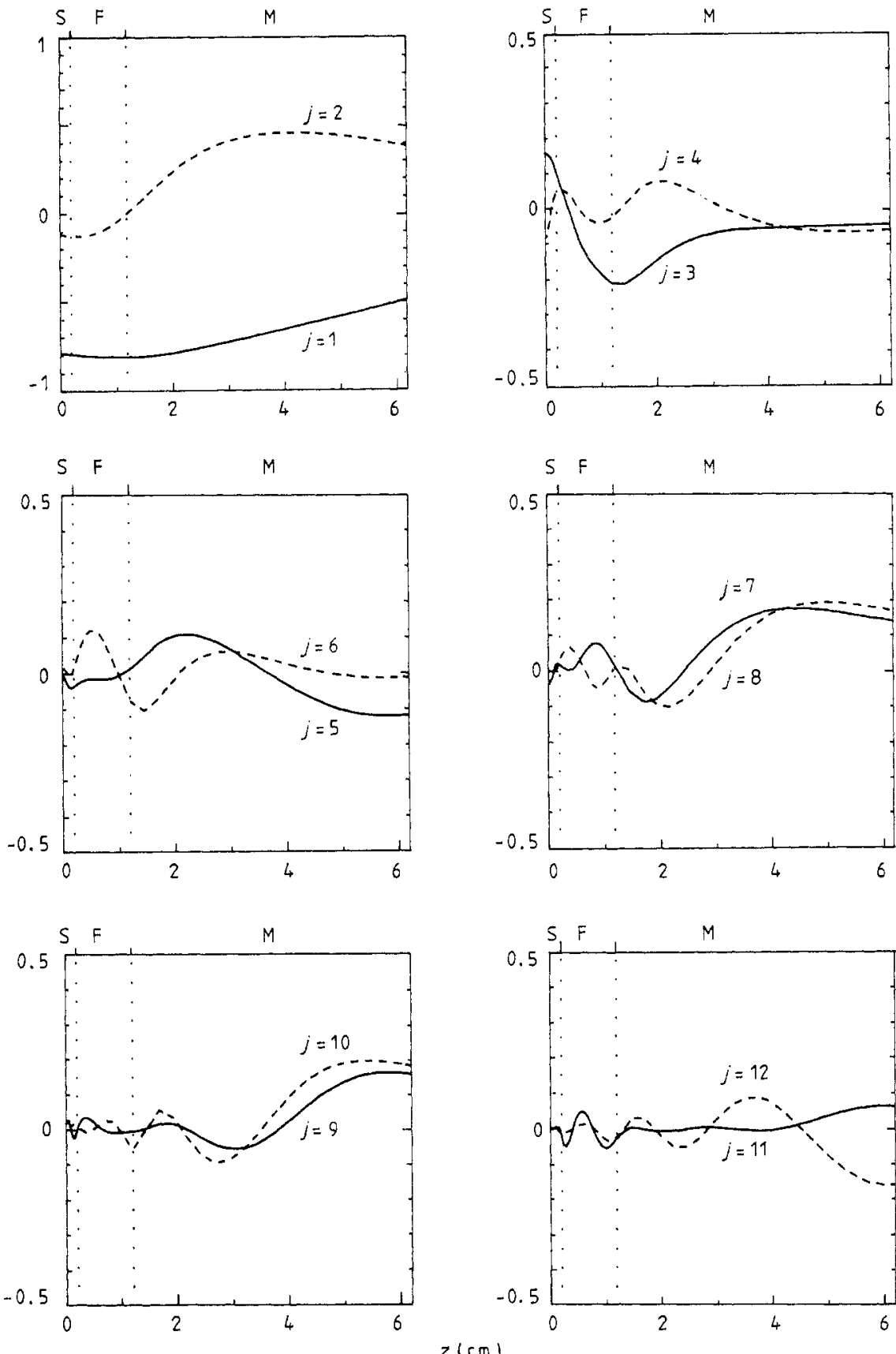

Figure 5. Continuous singular functions $\bar{u}_{N, j}$ against depth $z$, with $j=1, \ldots, 12$, for the SFM structure. 
The use of a space of continuous function makes it possible to take into account the information coming from measurement of the physical temperature at the surface of the body, $T(0)$. As discussed in $\S 1$, measurement of the skin temperature by means of infrared thermography has already been used for obtaining indications about the occurring internal processes.
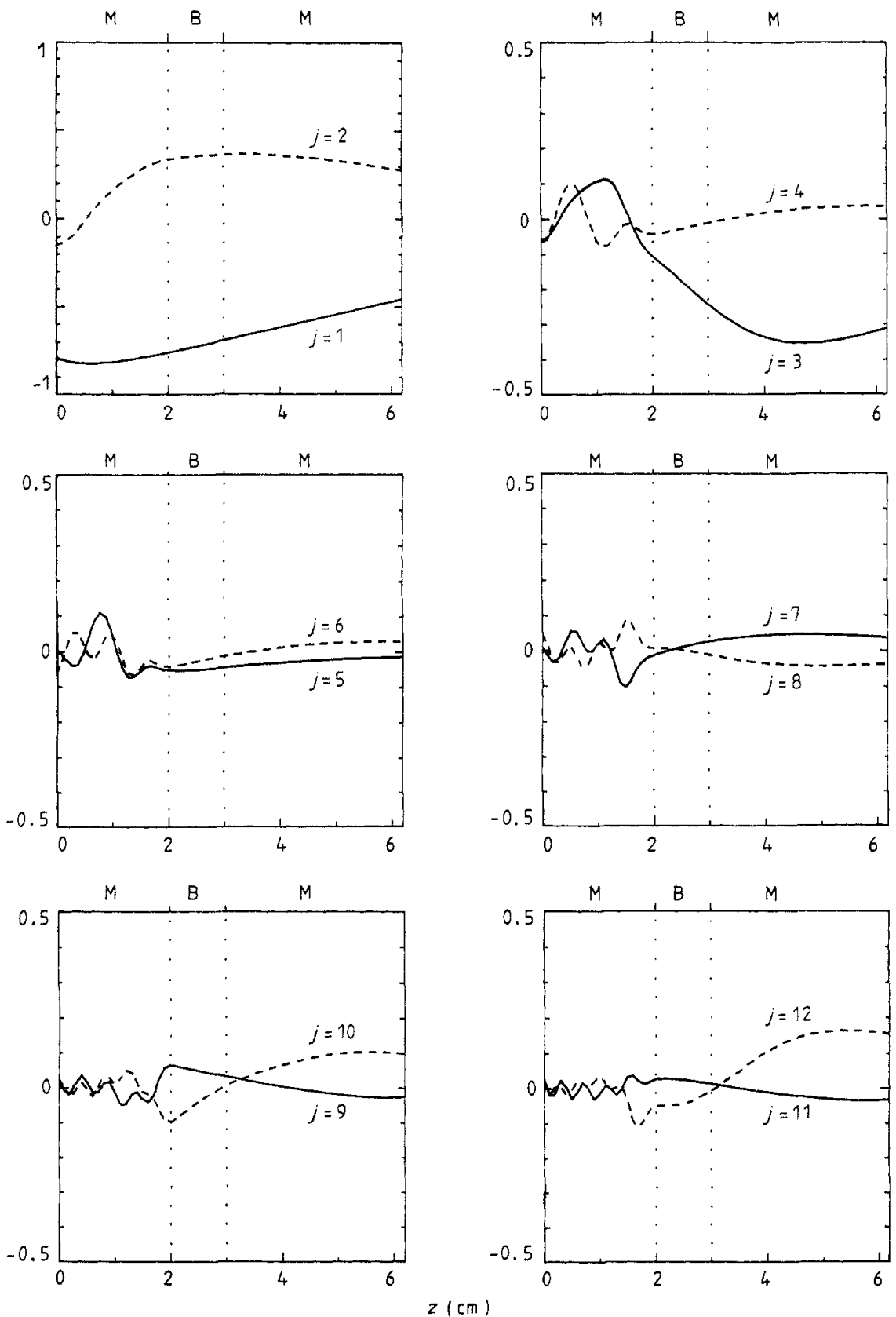

Figure 6. Continuous singular functions $\bar{u}_{N, j}$ against depth $z$, with $j=1, \ldots, 12$, for the MBM structure. 
In order to introduce such information in our formalism, we must express the surface temperature $T(0)$ as a functional in $\bar{X}$ of the temperature distribution $T(z)$, i.e. we must look for a function $\Phi_{0}(z)$ in $\bar{X}$ such that, for any $T(z)$, we have

$$
\left(T, \Phi_{0}\right)_{\bar{X}}=T(0)
$$

Considering again the case $K(z)=$ constant and $B(z)=0$, by means of a partial integration one derives from equation (4.12) that the second derivative of $\Phi_{0}$ is zero and that $\Phi_{0}$ must satisfy suitable boundary conditions. The result is

$$
\Phi_{0}(z)=\frac{1+h D}{2+h D}+\frac{h}{2+h D} z
$$

In order to take the value of the surface temperature into account, problem (4.6) must be extended by including also the case $n=0, g_{0}$ being the measured value of the surface temperature.

In table 4 we give the singular values of such a problem with 21 data (20 values of the brightness temperature in the band $1.5-6.5 \mathrm{GHz}$ plus the surface temperature). If we compare with table 3 , we see that we have just an increase of a unit in the number of singular values satisfying the condition $\bar{\alpha}_{N, 1} / \bar{\alpha}_{N, j}>10^{3}$.

In figures 7 and 8 we plot the singular functions $\bar{u}_{N, j}$ in the SFM and MBM structures, respectively.

Table 4. Singular values for the SFM and MBM structures, in the case of 20 equidistant data

\begin{tabular}{|c|c|c|c|c|}
\hline \multirow[b]{2}{*}{$j$} & \multicolumn{2}{|c|}{ SFM } & \multicolumn{2}{|c|}{ MPM } \\
\hline & $\bar{\alpha}_{N, j}$ & $\bar{\alpha}_{N, 1} / \bar{\alpha}_{N, j}$ & $\bar{\alpha}_{N, j}$ & $\bar{\alpha}_{N, 1} / \bar{\alpha}_{N, j}$ \\
\hline 1 & $3.65142 \times 10^{-1}$ & 1.0 & $4.00766 \times 10^{-1}$ & 1.0 \\
\hline 2 & $4.67340 \times 10^{-2}$ & 7.81321 & $4.65592 \times 10^{-2}$ & 8.60767 \\
\hline 3 & $1.50615 \times 10^{-2}$ & $2.42434 \times 10^{1}$ & $1.56204 \times 10^{-2}$ & $2.56566 \times 10^{1}$ \\
\hline 4 & $4.40429 \times 10^{-3}$ & $8.29060 \times 10^{1}$ & $3.13523 \times 10^{-3}$ & $1.27827 \times 10^{2}$ \\
\hline 5 & $8.29738 \times 10^{-4}$ & $4.40069 \times 10^{2}$ & $1.68579 \times 10^{-3}$ & $2.37732 \times 10^{2}$ \\
\hline 6 & $4.71801 \times 10^{-4}$ & $7.73932 \times 10^{2}$ & $1.33854 \times 10^{-3}$ & $2.99405 \times 10^{2}$ \\
\hline 7 & $9.21337 \times 10^{-5}$ & $3.96318 \times 10^{3}$ & $6.48639 \times 10^{-4}$ & $6.17856 \times 10^{2}$ \\
\hline 8 & $5.97890 \times 10^{-5}$ & $6.10718 \times 10^{3}$ & $5.23332 \times 10^{-4}$ & $7.65796 \times 10^{2}$ \\
\hline 9 & $2.89192 \times 10^{-5}$ & $1.26263 \times 10^{4}$ & $2.31534 \times 10^{-4}$ & $1.73092 \times 10^{3}$ \\
\hline 10 & $1.01397 \times 10^{-5}$ & $3.60110 \times 10^{4}$ & $1.97646 \times 10^{-4}$ & $2.02769 \times 10^{3}$ \\
\hline 11 & $4.51607 \times 10^{-6}$ & $8.08540 \times 10^{4}$ & $8.43116 \times 10^{-5}$ & $4.75339 \times 10^{3}$ \\
\hline 12 & $2.74443 \times 10^{-6}$ & $1.33049 \times 10^{5}$ & $3.42186 \times 10^{-5}$ & $1.17119 \times 10^{4}$ \\
\hline 13 & $7.09827 \times 10^{-7}$ & $5.14410 \times 10^{5}$ & $1.97101 \times 10^{-5}$ & $2.03331 \times 10^{4}$ \\
\hline 14 & $3.32353 \times 10^{-7}$ & $1.09866 \times 10^{6}$ & $6.78138 \times 10^{-6}$ & $5.90980 \times 10^{4}$ \\
\hline 15 & $2.60668 \times 10^{-7}$ & $1.40079 \times 10^{6}$ & $1.73866 \times 10^{-6}$ & $2.30503 \times 10^{5}$ \\
\hline 16 & $6.22980 \times 10^{-8}$ & $5.86122 \times 10^{6}$ & $8.39379 \times 10^{-7}$ & $4.77455 \times 10^{5}$ \\
\hline 17 & $4.10422 \times 10^{-8}$ & $8.89674 \times 10^{6}$ & $5.72604 \times 10^{-7}$ & $6.99901 \times 10^{5}$ \\
\hline 18 & $4.39715 \times 10^{-9}$ & $8.30406 \times 10^{7}$ & $3.24694 \times 10^{-7}$ & $1.23429 \times 10^{6}$ \\
\hline 19 & $2.17337 \times 10^{-99}$ & $1.68008 \times 10^{8}$ & $1.16084 \times 10^{-7}$ & $3.45238 \times 10^{6}$ \\
\hline 20 & $1.26361 \times 10^{-9}$ & $2.88968 \times 10^{8}$ & $1.86574 \times 10^{-8}$ & $2.14803 \times 10^{7}$ \\
\hline 21 & $7.33066 \times 10^{-10}$ & $4.98103 \times 10^{8}$ & $1.03749 \times 10^{-8}$ & $3.86283 \times 10^{7}$ \\
\hline
\end{tabular}
points in the band $1.5-6.5 \mathrm{GHz}$ plus the surface temperature. 


\section{The retrieval algorithm}

The solution of problem (3.6) is not unique, since any temperature distribution $T(z)$, which is orthogonal to the subspace spanned by the weighting functions $W_{n}(z)$, produces a zerodata vector. The same remark also applies to the problem (4.6), where the subspace is that
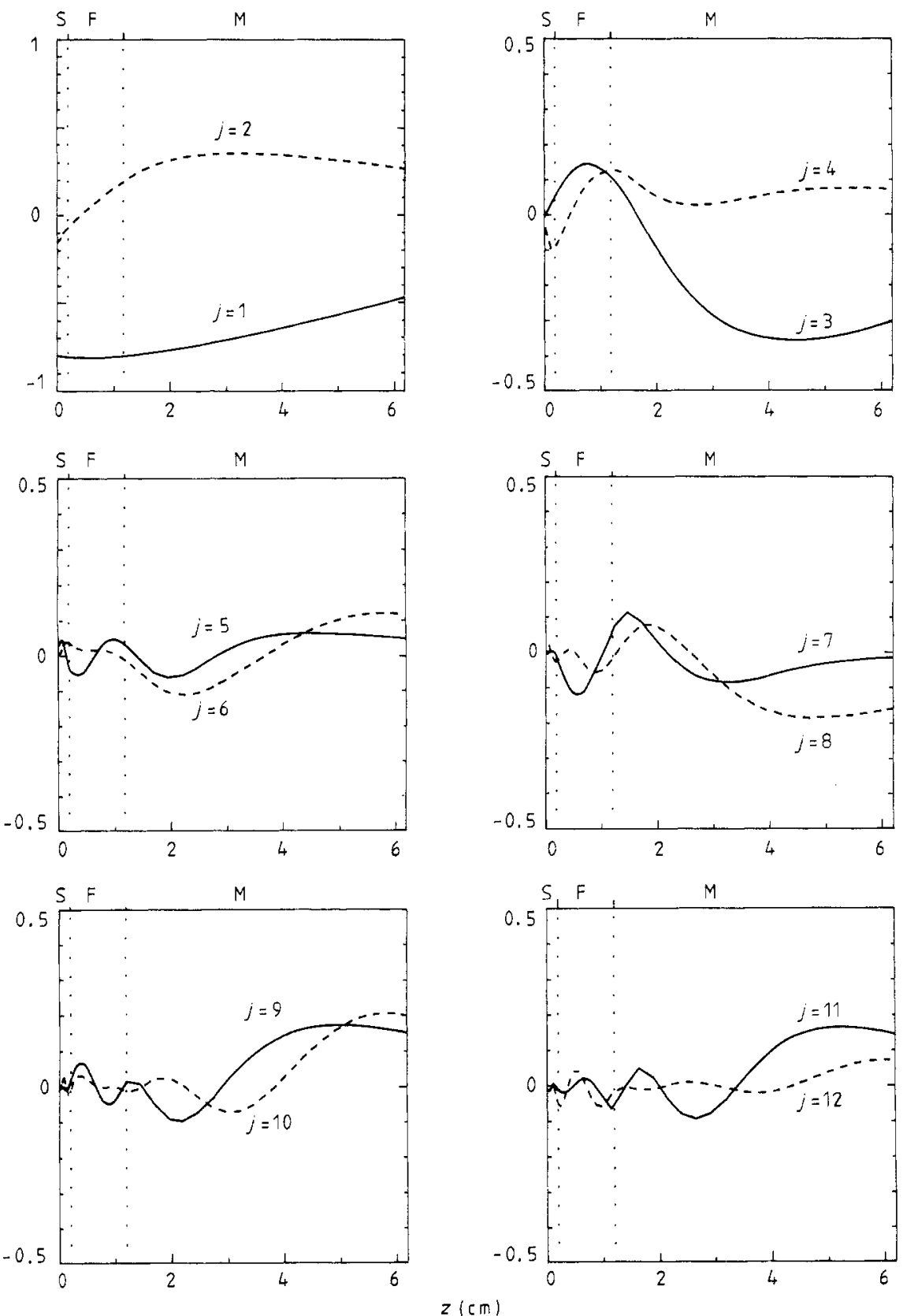

Figure 7. Continuous singular functions including surface temperature information $\bar{u}_{N, j}$ against depth $z$, with $j=1, \ldots, 12$, for the SFM structure. 
spanned by the functions $\Phi_{n}$ and orthogonality is intended in the sense of the scalar product (4.1). However, in both cases there exists a unique solution of minimal norm (normal solution), which is the unique solution in the subspace spanned by the functions $W_{n}(z)$ or $\Phi_{n}(z)$.
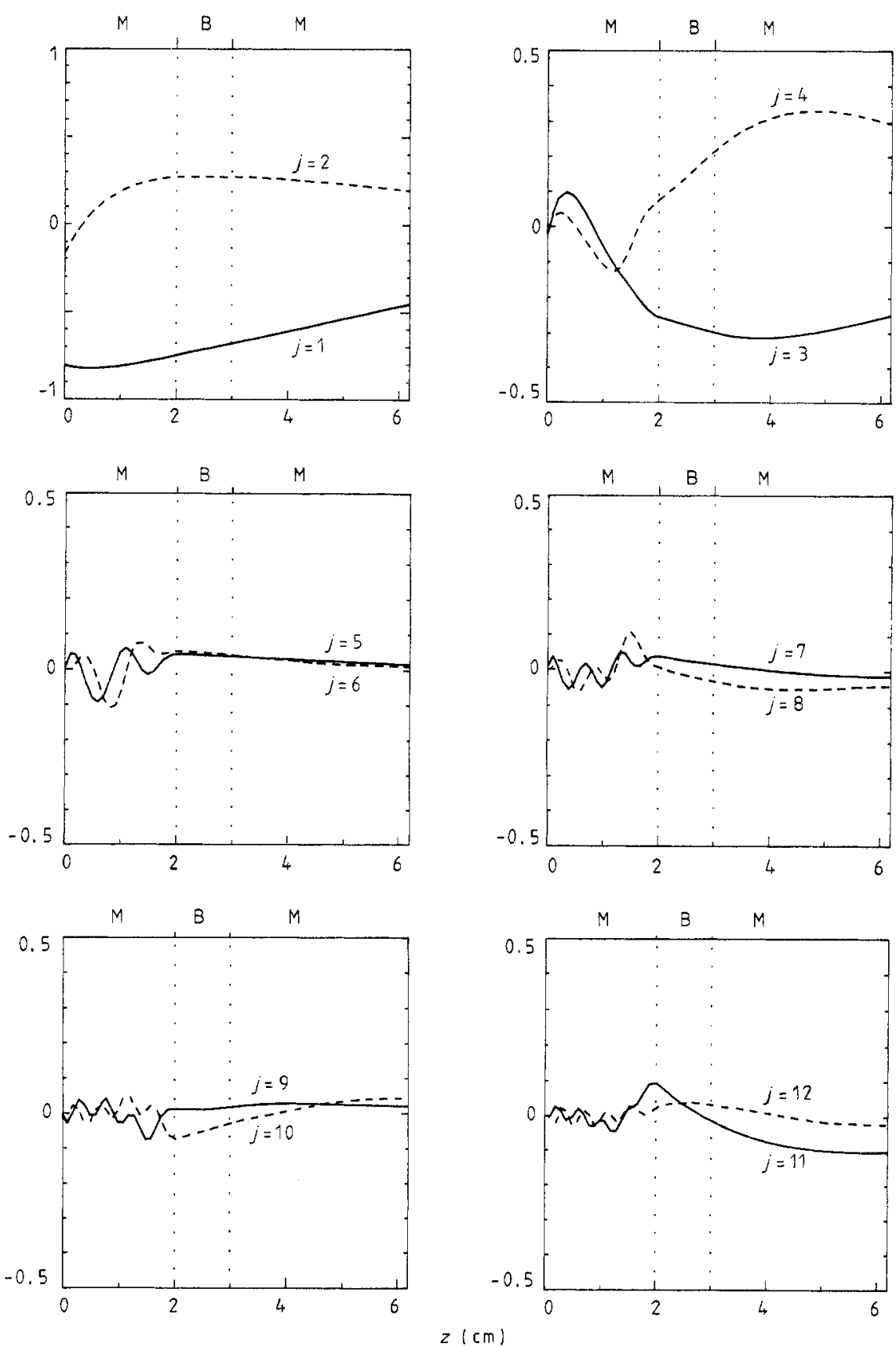

Figure 8. Continuous singular functions including surface temperature information $\bar{u}_{N, j}$ against depth $z$, with $j=1, \ldots, 12$, for the MBM structure. 
The normal solution can be expressed in terms of the singular system as follows [16]:

$$
T^{\dagger}(z)=\sum_{j=1}^{N} \frac{1}{\alpha_{N, j}}\left(\boldsymbol{g}, \boldsymbol{v}_{N, j}\right)_{Y_{N}} u_{N, j}(z) .
$$

In the case of problem (4.6) the same expression holds with the superscribed quantities.

The numerical stability of the solution (5.1) is controlled by the quantity $\alpha_{N, 1} / \alpha_{N, N}$, or $\bar{\alpha}_{N, 1} / \bar{\alpha}_{N, N}$ (see, e.g., $[14,16]$ ) which is called the condition number of the problem. Note that for the problem under consideration, with 20 data points, the condition number is quite large, ranging from $10^{6}$ to $10^{8}$ (see tables 1-4). Such an ill-conditioning is a consequence of the fact that problem (3.6) (and also problem (4.6)) is a discrete version of an ill-posed problem. Furthermore, by increasing the number of data points one gets enhanced ill-conditioning.

The condition number, however, may be a rather pessimistic estimate of error propagation from the data to the solution since it gives the 'worst' magnification of relative errors [13].

A more realistic estimate is given by the 'average magnification' of relative errors, $\beta^{\dagger}$, whose expression is [13]

$$
\beta^{\dagger}=\frac{1}{N}\left(\sum_{j=1}^{N} \alpha_{N, j}^{2}\right)^{1 / 2}\left(\sum_{j=1}^{N} \alpha_{N, j}^{-2}\right)^{1 / 2} .
$$

It is easily verified that this quantity is always smaller than $\alpha_{N, 1} / \alpha_{N, N}$ but greater than $1 / N$ $\left(\alpha_{N, 1} / \alpha_{N, N}\right)$. Therefore in our case this quantity is certainly larger than values ranging from $10^{5}$ to $10^{7}$ (condition number divided by 20 ). Also average error propagation is not acceptable.

From the previous remarks it follows that the normal solution is useless and that it necessary to introduce 'regularised' or 'filtered' solutions. In terms of singular function expansions, a rather general definition is the following:

$$
\tilde{T}(z)=\sum_{j=1}^{N} \frac{\xi_{j}}{\alpha_{N, j}}\left(\boldsymbol{g}, \boldsymbol{v}_{N, j}\right)_{Y_{N}} u_{N, j}(z)
$$

where the $\xi_{j}$ are suitable 'window coefficients' (or 'filter coefficients').

Tikhonov regularisation, for instance, gives a solution of this kind [22]. Another example is provided by truncated singular function expansions [14, 23], which correspond to a squared window, or by the use of a triangular window [24].

In all these cases we have a free parameter: the regularisation parameter in the case of the 'Tikhonov window' or the number $M$ of terms in the case of the squared and triangular windows. Therefore we need some criterion for the choice of such a parameter. This can be derived through a compromise between numerical stability of the solution and compatibility of the solution with the data.

In order to discuss this point, we assume a very simple probabilistic model for the data:

$$
g_{n}=\left(\mathscr{L}_{N} T\right)_{n}+\delta g_{n} \quad n=1, \ldots, N
$$

(we use the notation of $\S 3$ but all our considerations hold also in the case of the problem considered in $\S 4$ ) where the $\delta g_{n}$ are uncorrelated, signal-independent random variables, with zero expectation value and the same variance, $\varepsilon^{2}$ :

$$
\left\langle\delta g_{n}\right\rangle=0 \quad\left\langle\delta g_{n} \delta g_{m}\right\rangle=\varepsilon^{2} \delta_{n m} .
$$

Here the brackets denote expectation or ensemble average. 
Furthermore we also assume that $T(z)$ is from a random process such that the components of $T(z)$ with respect to the singular functions $u_{N, j}(z)$ are uncorrelated random variables, with zero expectation value and the same variance, $E^{2}$ :

$$
\left\langle\left(T, u_{N, j}\right)_{X}\right\rangle=0 \quad\left\langle\left(T, u_{N, m}\right)_{X}\left(T, u_{N, j}\right) X\right\rangle=E^{2} \delta_{m j}
$$

If we denote by $\mathrm{g}^{(0)}$ the noise-free data vector, i.e. $\mathrm{g}^{(0)}=\mathscr{L}_{N} T$, and by $\tilde{T}^{(0)}$ the corresponding filtered solution, then by means of computations similar to those performed in [13], we obtain

$$
\frac{\left\langle\|\delta \tilde{T}\|_{X}^{2}\right\rangle}{\left\langle\left\|\tilde{T}^{(0)}\right\|_{X}^{2}\right\rangle}=\beta^{2} \frac{\left\langle\|\delta g\|_{Y_{N}}^{2}\right\rangle}{\left\langle\left\|g^{(0)}\right\|_{Y_{N}}^{2}\right\rangle}
$$

where

$$
\begin{aligned}
& \beta=(1 / N \operatorname{Tr}[G])^{1 / 2}\left(\sum_{j=1}^{N} \frac{\tilde{\xi}_{j}^{2}}{\alpha_{N, j}^{2}}\right)^{1 / 2} \\
& \tilde{\xi}_{j}=\xi_{j}\left(\sum_{m=1}^{N} \xi_{m}^{2}\right)^{-1 / 2} .
\end{aligned}
$$

Note that, in the case $\xi_{j}=1(j=1, \ldots, N), \tilde{T}(z)$ coincides with the normal solution $T^{\dagger}(z)$ and the 'average magnification' $\beta$ coincides with $\beta^{\dagger}$, equation (5.2). This is in fact the maximum value of $\beta$ since the 'window coefficients' $\xi_{j}$ are always less than one. It is easy to see that if we decrease the regularisation parameter $\alpha$ in the 'Tikhonov window' or if we increase the number $M$ of terms in the squared and triangular windows, we get increasing values of the magnification parameter. In any case, for a given choice of the 'window coefficients', one can easily compute the 'average magnification' $\beta$ and get a precise estimate of error propagation.

The previous analysis gives a stability criterion for the choice of the filter. However, we must also satisfy another requirement, i.e. the compatibility of the solution with the data. To investigate this point one can introduce the so called discrepancy function which is the norm of the difference between the real data $g$ and the data computed from the filtered solution $\tilde{T}(z)$ :

$$
\rho(\boldsymbol{g})=\left\|\mathscr{L}_{N} \tilde{T}-g\right\|_{Y_{N}}^{2}
$$

By means of elementary computations one can derive the following relationship:

$$
\frac{\langle\rho(g)\rangle}{\left\langle\left\|g^{(0)}\right\|_{Y_{N}}^{2}\right\rangle}=\frac{1}{\operatorname{Tr}[G]} \sum_{j=1}^{N}\left(\xi_{j}-1\right)^{2} \alpha_{N, j}^{2}+\left(1 / N \sum_{j=1}^{N}\left(\xi_{j}-1\right)^{2}\right) \frac{\left\langle\|\delta \boldsymbol{g}\|_{Y_{N}}^{2}\right\rangle}{\left\langle\left\|g^{(0)}\right\|_{Y_{N}}^{2}\right\rangle} .
$$

The average discrepancy is zero when all the $\xi_{j}$ are equal to one, i.e. the filtered solution coincides with the normal solution. By comparing equation (5.11) with equation (5.8) we see that if we change the 'window' in order to reduce the magnification factor $\beta$ then we increase the average discrepancy and vice versa. Therefore the choice of the filter must be based on a compromise between error magnification and discrepancy.

In order to assess the quality of a retrieval algorithm, it is important to consider restorations of delta functions. Such a restoration indeed has the meaning of an impulse response of the system consisting of the radiometer plus the computer where the retrieval algorithm has been implemented. As a consequence the width of the main peak, corresponding to the delta function, can be interpreted as the resolution achievable with the used algorithm. 

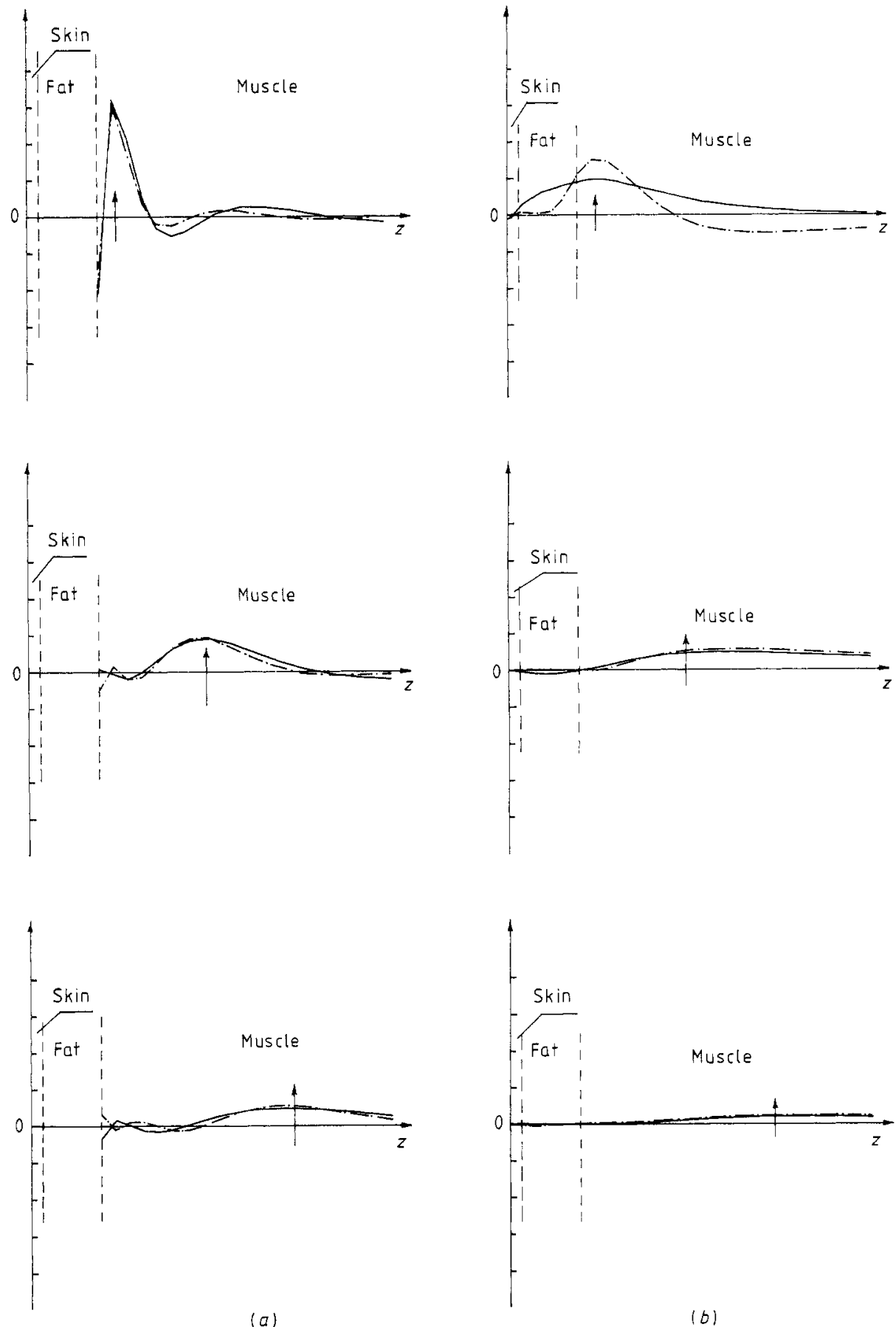

Figure 9. Retrieval of a delta function of temperature located at different depths in the muscle by $(a)$ discontinuous and $(b)$ continuous singular functions for the sFM structure. The full and chain curves refer to retrievals using squared or triangular window filters, respectively. The arrows on the horizontal frame lines indicate the location of the impulse to be retrieved, $(a) \longrightarrow, M=5, \beta=47 ;-\cdot-\cdot \cdot, M=6, \beta=50 ;(b)-, M=5, \beta=38$; -. - $., M=7, \beta=67$. 

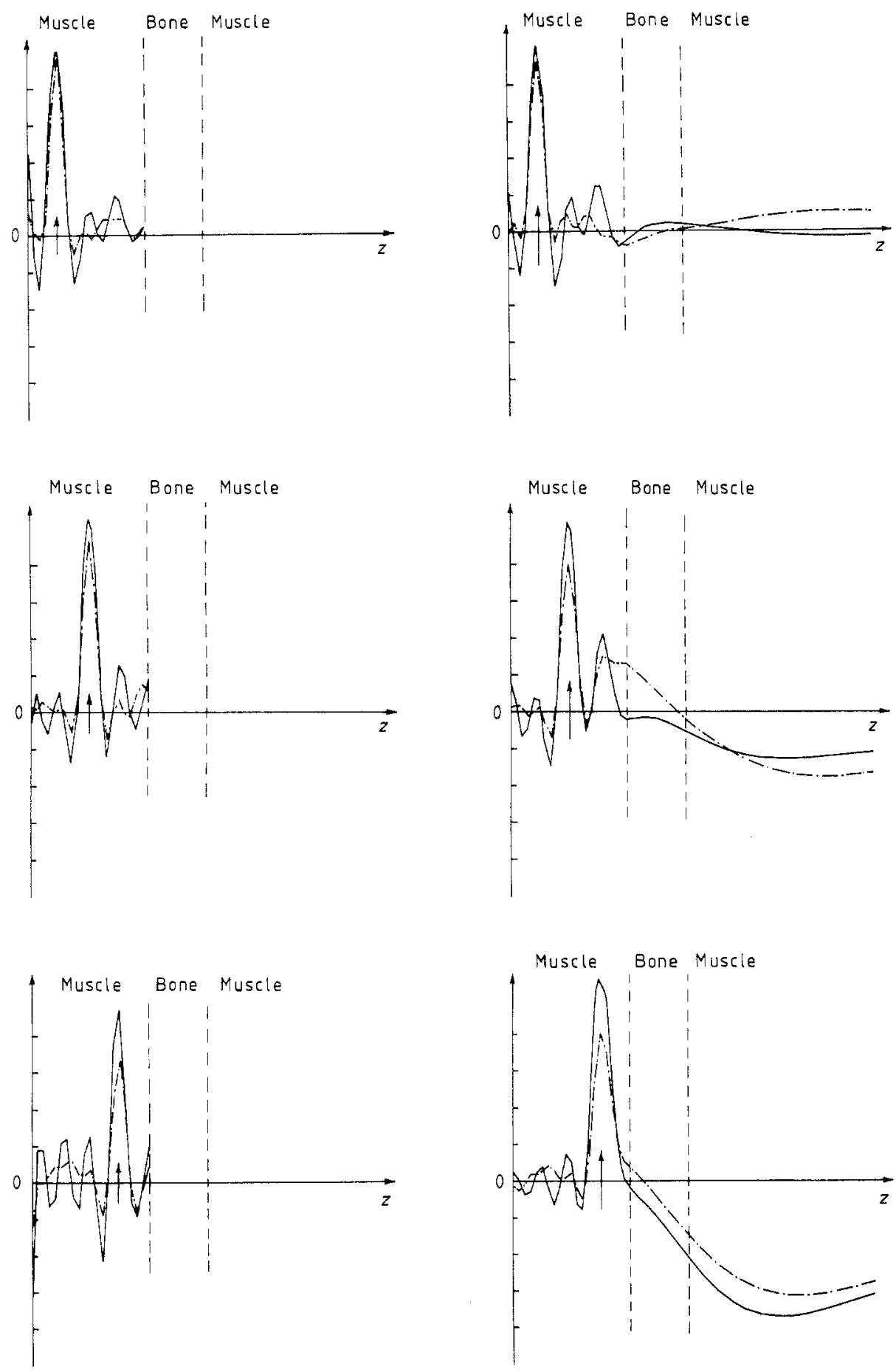

(a)

(b)

Figure 10. Retrieval of a delta function of temperature located at different depths in the outer $(0 \leqslant z \leqslant 2 \mathrm{~cm})$ muscle of the MBM structure. $(a)-, M=9 ; \beta=85 ;-\cdots-\cdots, M=14$, $\beta=116 ;(b) \longrightarrow, M=8, \beta=99 ;-\cdots,-, M=11, \beta=85$. 
We have performed several reconstructions of delta functions for the SFM and MBM structures both in the case of discontinuous and in the case of continuous singular functions, using both the squared window and the triangular window. The results are plotted in figures 9 and 10. It can be observed that the algorithm loses spatial resolution with increasing depth in the muscle for the SFM case, as argued from the analysis carried out in the preceding sections. On the contrary, only a slight loss of resolution with depth is observed for the MBM arrangement. Indeed, in this case the stationary wave pattern produced by the outer muscle-bone interface allows an overall spatial discrimination capability which is considerably better than in the SFM case and remains almost constant over the entire outer muscle. This trend is not altered appreciably either by the type (continuous or discontinuous) of singular functions or by the shape of the window used. The continuous singular functions which are needed for physically meaningful temperature retrievals in inhomogeneous discontinuous tissues lead to lower resolution with respect to the discontinuous functions, specially for the SFM case. On the other hand, the retrievals by continuous singular functions appear more stable than those by discontinuous ones.

\section{Conclusions}

The problem of the retrieval of thermal distributions in the human body from microwave radiometric measurements has been considered. The corresponding integral equations have been analysed in terms of singular function expansions. In this framework we have also investigated the effect of the additional measurement of the surface body temperature as may be obtained by infrared thermography. The results show that significant information can be extracted from the experimental data, especially in the case of muscular layers backed by low-water-content tissues.

Our analysis has been restricted to one-dimensional structures which, in many instances, are satisfactory models. Extensions to more realistic two- and three-dimensional models are in principle feasible, as appears from preliminary results that are being obtained in the analysis of scanning multispectral radiometry.

Use of the singular functions of the radiative integral operator is advantageous, since they are able to restore those features of the thermal pattern which contribute to the measured brightness temperatures. Special care in the measurements is also required in order to minimise the unstabilising effect of ill-conditioning. A relatively low number of measurement frequencies is beneficial not only in this respect, but also for reducing the overall complexity of the system, including data acquisition and computation time.

\section{References}

[1] Clark R P and Edholm O G 1984 Man and His Thermal Environment (London: Arnold)

[2] Gautherie M and Albert E (eds) 1983 Recent Advances in Medical Thermology (New York: Liss)

[3] Edrich J 1979 Centimeter and millimeter wave thermography. A survey on tumor detection J. Microwave Power 14 95-104

[4] Myers P C, Sadowsky N L and Barrett A H 1979 Microwave thermography: principles, methods and clinical applications J. Microwave Power 14 105-15

[5] Chive M, Plancot M, Giaux G and Prevost B 1984 Microwave hyperthermia controlled by microwave radiometry: technical aspects and first clinical results J. Microwave Power $19233-41$

[6] Miyakawa M 1981 Study on microwave thermography - application to the estimation of subcutaneous temperature profiles Trans IECE Japan E 64 786-92 
[7] Bardati F and Solimini D 1983 Radiometric sensing of biological layered media Radio Sci. 18 1393-401

[8] Westwater E R 1972 Ground-based determination of low-altitude temperature profiles by microwaves Mon. Wea. Rev. $10015-28$

[9] Hogg D C, Decker M T, Guiraud F O, Earnshaw K B, Merritt D A, Moran K P, Sweezy W B, Strauch R G, Westwater E R and Little C G 1983 An automatic profiler of the temperature, wind and humidity in the troposphere J. Climate Appl. Meteor. 22 807-31

[10] Smith W L 1983 The retrieval of atmospheric profiles from VAS geostationary radiance measurements J. Atmos. Sci. $402025-35$

[11] Bardati F and Solimini D 1984 On the emissivity of layered materials IEEE Trans. Geosci. Remote Sensing GE22 374-6

[12] Bertero M and De Mol C 1981 Ill-posedness, regularization and number of degrees of freedom Alti Fondazione G. Ronchi 36 619-32

[13] Twomey S 1974 Information content in remote sensing Appl. Opt. 13 942-5

[14] Pike E R, McWhirter J G, Bertero M and De Mol C 1984 Generalised information theory for inverse problems in signal processing IEE Proc. $131660-7$

[15] Miller G F 1974 Fredholm equations of the first kind Numerical Solution of the Integral Equations ed. L $M$ Delves and $\mathbf{J}$ Walsh (Oxford: Clarendon) p 175

[16] Bertero M, De Mol C and Pike E R 1985 Linear inverse problems with discrete data. I: General formulation and singular system analysis Inverse Problems $1301-30$

[17] Nashed M Z 1976 On moment discretization and least-squares solutions of linear integral equations of the first kind J. Math. Anal. Appl. 53 359-66

[18] Bertero M, Boccacci P and Pike E R 1982 On the recovery and resolution of exponential relaxation rates from experimental data: a singular-value analysis of the Laplace transform inversion in the presence of noise Proc. R. Soc. A 383 15-29

[19] Bertero M, De Mol C, Pike E R and Walker J G 1984 Resolution in diffraction-limited imaging, a singular value analysis. IV: The case of uncertain localization or non-uniform illumination of the object Opt. Acta $31923-46$

[20] Bertero M, Brianzi P and Pike E R 1985 On the recovery and resolution of exponential relaxation rates from experimental data: Laplace transform inversions in weighted spaces Inverse Problems 1 1-15

[21] Bardati F and Gerosa G 1983 An improved model describing the thermal behavior of electromagnetically irradiated living tissues Proc. Int. URSI Symp. on Electromagnetic Wave Theory, Santiago de Compostela (Universidad de Santiago de Compostela) pp 677-80

[22] Groetsch C W 1984 The Theory of Tikhonov Regularization for Fredholm Equations of the First Kind Research Notes in Math. No 105 (Boston: Pitman)

[23] Twomey S 1965 The application of numerical filtering to the solution of integral equations encountered in indirect sensing measurements J. Franklin Inst. 279 95-109

[24] Bertero M, Brianzi P, De Mol C, and Pike E R 1986 Positive regularized solutions in electromagnetic inverse scattering Proc. Int. URSI Symp. on Electromagnetic Theory, Budapest (Budapest: Akadémiai Kiadó) pp 150-2 